\title{
New Method for Millimeter-Wave Signal High Sensitivity Receiving and Testing in Range
}

\author{
X. Chen and J. Z. Xu
}

\begin{abstract}
Based on the actual demand of the weak signal (low to -128 dBm) detection in millimeter-wave range test, and through the analysis of signal-to-noise ratio, the $\mathrm{W}$-band receiver system of $\mathbf{- 1 2 8} \mathrm{dBm}$ sensitivity is achieved by using the technology of super-heterodyne broadband multi-channel fast swept-frequency composite channelization. With the designed millimeter-wave calibration source and the spatial attenuation theory, a new test method of the high sensitivity $(-128 \mathrm{dBm})$ in W-band is put forward. Theoretical analysis shows that the minimum power of the millimeter-wave signal source can reach $-128 \mathrm{dBm}$ in $\mathrm{W}$-band when the test distance is $290 \mathrm{~m}$ and the corresponding attenuation value is about $-121.5 \mathrm{~dB}$. The practical measurements using the calibrated millimeter-wave signal source demonstrate the feasibility of the proposed test method.
\end{abstract}

Index Terms-Range test, high sensitivity, millimeter-wave source, spatial attenuation, weak signal, $\mathrm{W}$-band, signal to noise interference ratio.

\section{INTRODUCTION}

With the rapid development of millimeter-wave weapons, the research on the millimeter-wave test equipments and the technology of range is vigorously promoted to realize the range millimeter-wave signal monitoring and qualification test of weapons systems [1]-[4]. Due to the measured target in the range test often in motion and some missile-borne millimeter-wave measurement systems working in passive state, the weak millimeter-wave signal requires high sensitivity receiver in the testing system. The advanced level for W-band test system in the world can achieve $-128 \mathrm{dBm}$ [5]. But as a result of technical blockade and equipment embargo in the high-end research field, the range millimeter-wave testing technique gap between our country and the western countries is still big, we only reach the sensitivity of $-120 \mathrm{dBm}$ W-band high sensitivity in the receiver system [6]. Meanwhile, for the lack of the millimeter-wave band (especially W-band) test instrument, there is no testing standards and conditions for more than 40 $\mathrm{GHz}$ system performance [7], and there exist no performance test and calibration of the high-sensitivity W-band system in our country.

In order to make a breakthrough in W-band test field, this paper, by using weak signal detection technology of super-heterodyne broadband multi-channel fast sweptfrequency composite channelization and the low noise amplifier good in W-band noise coefficient, develops a

Manuscript received December 18, 2012; revised March 10, 2013

The authors are with the School of Electronic Engineering and Optoelectronic Technology, Nanjing University of Science and Technology, Nanjing, 210094 China (e-mail: 10cx14@163.com,xjz2018@yeah.net ).
W-band test system with the sensitivity to $-128 \mathrm{dBm}$. And in the combination of the millimeter-wave calibration source with the spatial attenuation method, a minimum $-128 \mathrm{dBm}$ W-band millimeter-wave weak signal source is provided, realizing the test and calibration on the sensitivity of receiving system.

\section{Detection of Millimeter-Wave Weak Signal}

\section{A. Detection Method}

The key of weak signal detection is to eliminate the noise and improve the sensitivity of signal detection, namely to improve signal to noise interference ratio (SNIR) of the detection system. Noise is the basic reason for the sensitivity limit of the receiver. For the receiver noise coefficient, the primary design is very important, so in order to effectively improve the receiving sensitivity, the low noise amplifier (LNA) is placed in the front of the receiver. According to the current millimeter-wave components level, the noise coefficient of a single W-band millimeter-wave LNA is 6-9 $\mathrm{dB}$, the gain is $20 \mathrm{~dB}$. And the noise coefficient of the W-band mixer is 6-8 dB [8]. Take the two stage cascade of LNA and a single stage of the mixer into consideration, the total noise coefficient is [9]:

$$
F=F_{1}+\frac{F_{2}-1}{G_{1}}+\frac{F_{3}-1}{G_{1} G_{2}}+\frac{F_{4}-1}{G_{1} G_{2} G_{3}}
$$

where $F_{1}$ is the noise coefficient for radio frequency transmission loss (take $2 \mathrm{~dB}$ ); $G_{1}$ is the gain caused by the radio frequency loss in the transmission line (take $-5 \mathrm{~dB}$ ), $F_{2}$ is the noise coefficient of the first stage LNA (take $7 \mathrm{~dB}$ ), $G_{2}$ is the gain of the first stage LNA (take $20 \mathrm{~dB}$ ), $F_{3}$ is the noise coefficient of the second stage LNA (take $8 \mathrm{~dB}$ ), $G_{3}$ is the gain of the second stage LNA (take $20 \mathrm{~dB}$ ), $F_{4}$ is the noise coefficient of the first stage mixer (take $7 \mathrm{~dB}$ ). As shown in (1), the total noise coefficient is $11.6 \mathrm{~dB}$. We can see that the system noise coefficient is mainly influenced by the radio frequency transmission loss and the millimeter-wave LNA, so in order to effectively reduce the noise coefficient, the W-band LNA with the current international leading noise coefficient index is used.

The concept of sensitivity originates from the minimum detectable signal power in the radar equation, namely the least input signal power when the signal to noise ratio is equal to 1 . The higher the receiver sensitivity, the stronger the weak signal reception ability is. The system sensitivity of the receiver can be calculated in the formula as follows [10]:

$$
P_{S \text { min }}=k T_{o} B_{R I} F
$$


where $k$ is Boltzmann constant $\left(1.38 \times 10^{-23} \mathrm{~J} / \mathrm{k}\right), T_{o}$ is the receiver's operating temperature (take $290 \mathrm{~K}$ ), $B_{R I}$ is the linear pass-band of the receiver (the total pass-band of high frequency and intermediate frequency part of receiver). If the measured signal is very weak, we can reduce the pass-band bandwidth of the receiver to improve the sensitivity of the system. Therefore according to the calculation as shown in (2), the pass-band bandwidth of the receiver should be smaller than $3 \mathrm{kHz}$ to meet the requirements of receiving the $-128 \mathrm{dBm}$ minimum signal.

However, the actual pass-band bandwidth of the received signal in $\mathrm{W}$-band is $6 \mathrm{GHz}$. To improve the sensitivity of the system receiver, the channel processing technology is used. And by taking the margins into account, the minimum resolution bandwidth of the channelized receiver is designed to $1 \mathrm{kHz}$. But the amount of $6 \times 10^{6}$ channels is needed when the $6 \mathrm{GHz}$ bandwidth is transformed into the $1 \mathrm{kHz}$, which is quite difficult in engineering realization. Taking it into account that the measured signals have a certain dwell time, the time for space approach is used, which means to use the heterodyne method to shift the broadband signal into the intermediate frequency band, and then mix the measured signal with the swept-frequency synthesizer and divide the wider broadband into a set of sub-band with narrow bandwidth in time. By doing so, the number of channels can be greatly reduced and still achieve $1 \mathrm{kHz}$ channel bandwidth after the two stages processing.

Due to the dynamic test speed requirements and the large signal dispersion bandwidth, the direct digital frequency synthesizer (DDS) swept-frequency and channelization receiving are combined in the treatment. The processes are as follows: divide $1 \mathrm{GHz}$ dispersion bandwidth of the measured signal into $200 \mathrm{MHz}$ sub-band by using the swept-frequency synthesizer, and each $200 \mathrm{MHz}$ sub-band is divided into 10 parallel $20 \mathrm{MHz}$ channels through channelization, each of which, by the phase-locked loop (PLL) frequency synthesizer, is frequency-swept, by step with $1 \mathrm{MHz}$, to achieve parallel 20 point/channel frequency sweeping. In order to improve the channelization processing speed further, each $1 \mathrm{MHz}$ frequency point is tested with $1 \mathrm{kHz} / \mathrm{step}$ by the method of fast swept-frequency of DDS and the crystal filter group is used to complete fine narrow-band filtering to reduce the bandwidth of the intermediate frequency receiver to $1 \mathrm{kHz}$, ensuring the detection sensitivity. With the rapid feature of DDS, the swept-frequency time for each $1 \mathrm{kHz}$ within $1 \mathrm{MHz}$ is reduced to less than $1 \mu \mathrm{s}$, making the response speed of the channelized receiver better than $50 \mathrm{~ms} /$ $200 \mathrm{MHz}$.

\section{B. Analysis of Signal to Noise Interference Ratio}

The signal to noise interference ratio of the weak signal detection system is equivalent to the ratio of input noise bandwidth to the system noise equivalent bandwidth. The larger the SNIR, the stronger the noise processing ability and the higher the weak signal detection level.

When the radiation resistance of the antenna and the input resistance of the receiver matches, the total input noise power can be calculated according to the corresponding relation between the power and the temperature:

$$
P_{n}=k\left(T_{e}+T_{o}\right) B=k T_{o} F B
$$

where $T_{e}=(F-1) T_{o}$ is equivalent noise temperature. The minimum input signal level $P_{i n}=-128 \mathrm{dBm}$, so the input SNR of the receiver is,

$$
I_{\mathrm{SNR} \_ \text {in }} \geq P_{\text {in }} / P_{n}=-63.4 \mathrm{~dB}
$$

Considering the back-end signal processing (such as the radio frequency measurement in passive monitoring), we take $I_{\text {SNR_out }}=4 \mathrm{~dB}$, according the definition, SNIR of the whole system should satisfy $I_{\mathrm{SNIR}} \geq 67.4 \mathrm{~dB}$.

It is assumed that the receiver noise obeys the normal distribution (Gaussian white noise), according to the analysis of section $\mathrm{A}$, the input noise bandwidth is approximate as the radio frequency bandwidth $\mathrm{B}=6 \mathrm{GHz}$, and the minimum resolution of the channelized receiver is $1 \mathrm{kHz}$ bandwidth, so the maximum SNIR provided by the channelized receiver can be expressed as:

$$
I_{\text {SNIR_max }}=\frac{B}{\Delta f_{\min }}=67.8 \mathrm{~dB}
$$

From (5), we can see that it can improve SNR by $67.8 \mathrm{~dB}$ through the weak signal detection method of superheterodyne broadband multi-channel fast swept-frequency composite channelization, realizing the reliability of the W-band weak signal $(-128 \mathrm{dBm})$ detection and the demodulation of the corresponding parameters.

\section{The System Sensitivity TeSt Method}

\section{A. Test Principle}

The system receiver sensitivity is a comprehensive index. If the system meets the demands of the receiver sensitivity index, it is implied that other indicators of the system also meet the index requirements. At present, the system sensitivity test method is to add the known standard power source of the precise attenuation to the receiver input for a test in the laboratory environment [11], [12]. But the high-precision attenuators and the good performance isolators are lacked in W-band, so the leakage power of the signal source is larger, which is close to or even larger than the output energy of the attenuator. Besides, according to the experience from the indoor measurement, the attenuation effect is decreased obviously when the attenuation value is more than $90 \mathrm{~dB}$, namely the millimeter-wave signal will be no longer weakened with the increase of the attenuation value. Therefore, to test the sensitivity index of $-128 \mathrm{dBm}$ of the $\mathrm{W}$-band receiver system, the key is to provide a $\mathrm{W}$-band millimeter-wave weak signal source with its minimum power of $-128 \mathrm{dBm}$.

Based on the design and development of the millimeterwave calibration source, the sensitivity test experiment plan of the receiver is designed under the existing condition according to the spatial distance attenuation principle. The test principle is shown in Fig.1. The method uses the 
millimeter-wave calibration source after spatial distance attenuation assisted by the absorbing materials with a certain attenuation value to realize the $-128 \mathrm{dBm}$ weak millimeterwave power signal, which is required by the measurement of millimeter-wave signal monitoring system.

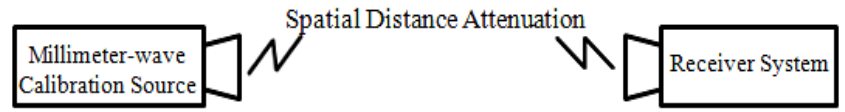

Fig. 1. Sensitivity testing principle

Provided the power of the emission source is $P_{t}$, the gain of the antenna is $G_{t}$, then the power intensity at a distance of $R$ to the emission source along the wave beam axis is,

$$
I_{s}=P_{t} G_{t} / 4 \pi R^{2} \quad\left(\mathrm{~W} / \mathrm{m}^{2}\right)
$$

When the signal is received by the isotropic antenna, and the effective aperture area of which is $A_{e}=\lambda^{2} / 4 \pi\left(\mathrm{m}^{2}\right)$, the power received is as follows,

$$
P_{r}=P_{t} G_{t} \lambda^{2} /\left(4 \pi R^{2}\right) \quad(\mathrm{W})
$$

where $\lambda$ is the operating wavelength.

We can see that the spatial distance attenuation $L_{R}$ caused by distance $R$ is:

$$
L_{R}=\lambda^{2} /\left(4 \pi R^{2}\right)
$$

When the operating wavelength $\lambda=3 \mathrm{~mm}$ (in W-band), the influence of atmosphere attenuation is ignored, the relationship between the spatial distance attenuation $L_{R}$ and the distance $R$ is showed in Fig. 2 .

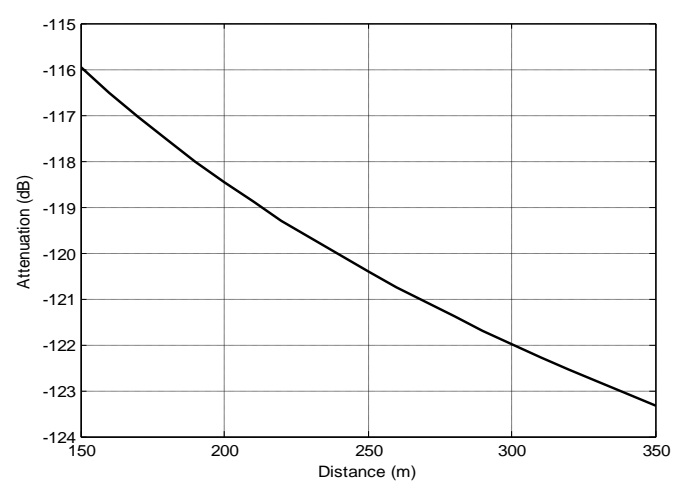

Fig. 2. Relationship between spatial attenuation and distance

From Fig. 2, we can see that the value of spatial distance attenuation in $\mathrm{W}$-band probably range from -116 to $-123 \mathrm{~dB}$ when the distance range from 150 to $350 \mathrm{~m}$, which satisfy the condition of far less than $-90 \mathrm{~dB}$.

\section{B. Millimeter-Wave Calibration Source}

The calibration source consists of the millimeter-wave power source, the standard antenna, the fixed attenuator, the multilayer absorbing materials and the power supply. Its simplified structure is shows in Fig.3. The import W-band standard source is used as the millimeter-wave power source, and its operating frequency is selected in the middle of receiver band of the measured millimeter-wave signal monitoring system with its power magnitude $P_{t}$ ranging from $0-20 \mathrm{dBm}$. The millimeter-wave antenna is a standard horn antenna, the gain is $G_{t} \approx 20 \mathrm{~dB}$. The fixed attenuator is the standard broadband fixed attenuator usually with the value of attenuation $L_{D}=30-40 \mathrm{~dB}$. The millimeter-wave power source signal first radiates through the antenna radiation, then passes through absorbing material and distance attenuation, and finally arrives at the receiver antenna aperture of the millimeter-wave signal monitoring system. We can adjust the attenuation value $L_{M}$ to meet the requirements of the test sensitivity in the tested system by using the absorbing plate, and increasing or decreasing the thickness or number of the absorbing plate.

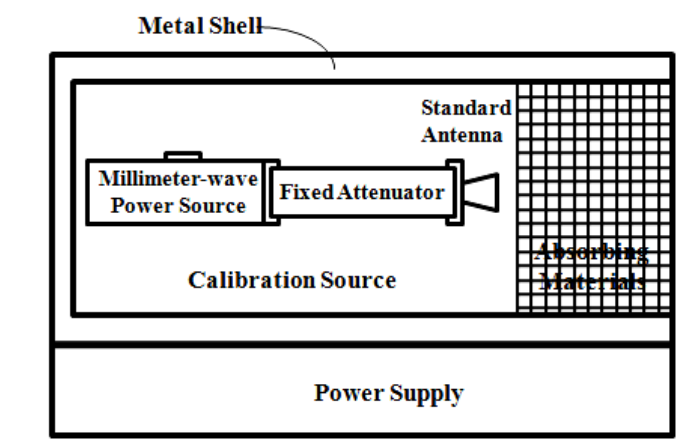

Fig. 3. Structure diagram of the millimeter-wave calibration source

\section{EXPERIMENT AND RESULT}

Based on the above method, the concrete steps of the receiving sensitivity test of the millimeter-wave signal monitoring system are as follows:

1) The millimeter-wave calibration source should be calibrated in the laboratory environment. Specifically, the millimeter-wave frequency meter and the power meter are respectively used to measure the frequency $f_{0}$ and the power $P_{t}$ of the millimeter-wave power source. The attenuation value $L_{M}$ of the absorbing material is measured by the actual power source and the power meter in the anechoic chamber. The gain $G_{t}$ of the millimeter-wave standard antenna and the attenuation $L_{D}$ of the fixed attenuator are provided by the suppliers, which are determined according to actual operating frequency. The parameters of the millimeter-wave calibration source after the first calibration are showed in Table I.

TABLE I: PARAMETERS OF THE MILLIMETER-WAVE CALIBRATION SOURCE

\begin{tabular}{llllll}
\hline \hline \multirow{2}{*}{ Parameters } & $f_{0}$ & $P_{t}$ & $L_{D}$ & $G_{t}$ & $L_{M}$ \\
& $(\mathrm{GHz})$ & $(\mathrm{dBm})$ & $(\mathrm{dB})$ & $(\mathrm{dB})$ & $(\mathrm{dB})$ \\
\hline W-band & 94.53 & 10.0 & -30.0 & 19.5 & -32.9 \\
\hline \hline
\end{tabular}

2) According to the calibration value of the parameters of the millimeter-wave calibration source, the choice of the testing distance in field experiment can be obtained by the analysis and calculation. The known receiver power of the receiver system is equivalent to,

$$
P_{A}(\mathrm{dBm})=P_{t}+L_{D}+G_{t}+L_{M}+L_{R}+G_{r}
$$


where, the receiving antenna gain $G_{r}=26 \mathrm{~dB}$ (average value of the measured data). In order to make the millimeter-wave signal power arriving at the antenna aperture of the measured millimeter-wave signal monitoring system satisfy the sensitivity requirement of $-128 \mathrm{dBm}$, by putting the parameters in Table 1 into (9) and considering (8) or Fig.2, it can be obtained that when the testing distance is $290 \mathrm{~m}$, the value of the spatial distance attenuation $L_{R}$ is about to be $-121.5 \mathrm{~dB}$, then the received power of receiver is -128.9 $\mathrm{dBm}$.

3) To master the free space test field. According to step 2), the measured millimeter-wave signal monitoring system and the millimeter-wave calibration source should be placed at the position apart from $290 \mathrm{~m}$, and the actual test experiment is conducted at the stadium of Nanjing University of Science and Technology shown as Fig.4. The measured millimeterwave signal monitoring system is placed in the second floor platform, and the millimeter-wave calibration source is placed in the round platform which is in the opposite of the second floor platform with the similar vertical height, or vice versa. The test distance is adjusted based on the actual measurement and satellite photos ranging. In order to guarantee the calibration accuracy, the testing environment is clear, no wind, $20^{\circ} \mathrm{C}$ with no metal objects, buildings, trees and other obstacles higher than the venue plane within the test distance range and $30 \mathrm{~m}$ at both sides.

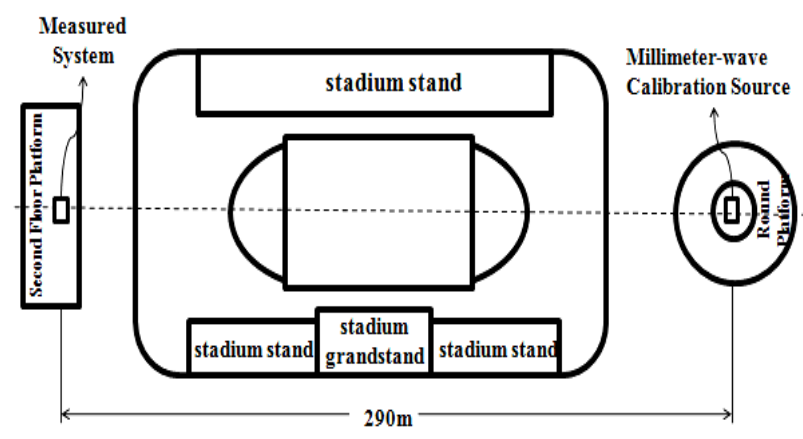

Fig. 4. Station diagram of system sensitivity test

4) According to step 1) to step 3), the system sensitivity is measured repeatedly under the test conditions. The measured results show that the average field intensity arriving at the antenna aperture of the receiver is $-128 \mathrm{dBm}$.

\section{CONCLUSION}

Based on the actual needs of the millimeter-wave weapons test systems in the national shooting range, researches is made on the method of the reception and sensitivity measurement of the millimeter-wave weak signal (as low as $-128 \mathrm{dBm}$ ). On the basis of the analysis on signal-to-noise ratio, the technology of super-heterodyne broadband multi-channel fast swept- frequency composite channelization is adopted, and the $\mathrm{W}$-band receiver system of $-128 \mathrm{dBm}$ sensitivity is achieved, which, a breakthrough in the existing W-band test means in our country, can reliably detect the range millimeter-wave weak signals and demodulate the corresponding parameters.

In terms of the problem that there is no condition of the sensitivity testing of the W-band system with high sensitivity, a new method of the high sensitivity testing is proposed. The core is to use the spatial attenuation theory and the self-developed millimeter-wave calibration source to provide a millimeter-wave standard signal source in $\mathrm{W}$-band with the power as low as $-128 \mathrm{dBm}$. The feasibility of the method is proved by the actual measurements in the free space test field, and the method serves as a new means for the range test equipments and technology.

But in order to the easy implementation of the high sensitivity receiver, the critical low-noise amplifier module in the front of the receiver is still used the imported devices, which is not conducive to reduce costs in engineering. So the design of $\mathrm{W}$-band broadband low-noise amplifier will be conducted in further studies.

\section{ACKNOWLEDGMENT}

This work is partially supported by the China Postdoctoral Science Foundation Funded Project (No. 20100481151). The authors also gratefully acknowledge the helpful comments and suggestions of the reviewers, which have improved the presentation.

\section{REFERENCES}

[1] M. M. Sayed, "Millimeter wave tests and instrumentation," presented at the 65th ARFTG Conference Digest, June 17, 2005.

[2] W. Wang, P. Xu, and D. F. Lin, "The present situation and development trend for guided weapons range test semi-physical simulation," Aerodynamic Missile Journal, vol. 5, pp. 11-15, 2012.

[3] F. R. Ji, Y. Q. Xu, and L. Ji, "Design of detection system for complex artillery weapon communication system," in 2011 Intelligent Computation Technology and Automation, Shenzhen, 2011, pp 411-414.

[4] S. W. Harmer, N. Bowring, D. Andrews, N. D. Rezgui, M. Southgate, and S. Smith, "A review of nonimaging stand-off concealed threat detection with millimeter-wave radar," IEEE Trans. on Microwave Magazine, vol. 13, no. 1, pp. 160- 167, Jan.-Feb. 2012.

[5] M. Khanpour, K. W. Tang, P. Garcia, and S. P. Voinigescu, "A wideband W-band receiver front-end in 65-nm CMOS," Solid-State Circuits, vol. 43, no. 8, pp. 1717- 1730, Aug. 2008.

[6] X. W. Gu, "Design of millimeter-wave high-sensitivity receiver," M.S. thesis, Dept. Electron. Eng. and Optoelectron. Tech., NUST, Nanjing, China, 2008

[7] $18 \mathrm{GHz}-40 \mathrm{GHz}$ Attenuation Standard Device [S/OL]. (July 26, 2011) National Institute of Metrology P. R. China. General Administration of Quality Supervision, Inspection and Quarantine of the People's Republic of China. [Online]. Available: http://www.nms.org.cn/Resource/PrimaryItem.aspx?id=498.

[8] P. Zhou, P. Zheng, W. H. Yu, and H. J. Sun, "Design of a W-band low noise amplifier module with MMIC," in 5th Global Symposium Millimeter Waves, Beijing, 2012, pp. 5-8.

[9] Y. C. Chang, S. G. Lin, H. K. Chiou, D. C. Chang, and Y. Z. Juang, "On-wafer noise figure measurements of millimeter-wave LNA and mixer," in 2010 Asia-Pacific Microwave Conference Proceedings, Yokohama, 2011, pp. 1424-1427

[10] B. Stec, "Sensitivity of broadband microwave receiver with consideration nonlinear part," in the 15th International Conf. Microwaves, Radar and Wireless Communications, 2004, vol. 3, pp. 940-943.

[11] H. J. Hansen, "The detection of W-band (94 GHz) millimetric wave emitters," in 2000 Asia-Pacific Microwave Conference, Sydney, 2002, pp. 469-473.

[12] T. Y. Wu and S. W. Chua, "A millimeter-wave attenuation measurement standard from $26.5 \mathrm{GHz}$ to $40 \mathrm{GHz}$," in 2009 European Microwave Conference, Rome, 2009, pp. 1603-1606. 


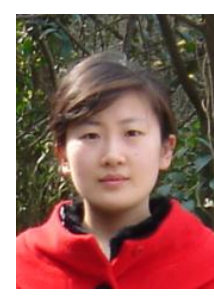

Xi Chen was born in Xuzhou, China, in 1984.

She received the B.S. degree in

Communication Engineering from North

University of China, Taiyuan, China, in 2007.

She is currently working toward the Ph.D.

degree in Communication and Information

Systems in the School of Electronic and

Optical Engineering, Nanjing University of Science and Technology, Nanjing, China.

Her current research interests involve millimeter- wave range testing technology and signal processing.

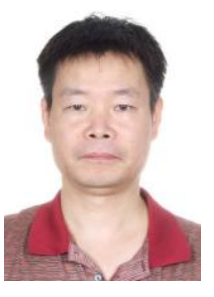

Xujian Zhong was born in Wuxi, China, in 1958. He received the M.S. degree in Electronic Engineering from Nanjing University of Science and Technology (NUST), Nanjing, China, in 1982.

Since 1982, he worked with School of Electronic and Optical Engineering of NUST in previous positions of vice director of Short Range and Detection Technical Office, vice director of Applied Electronic Technical Department, and director of Electronic System Office. He was with Bauman Moscow State Technical University (BMSTU) as a senior visiting scholar in 2002 and 2003. He is currently a professor of School of Electronic Engineering and Optoelectronic Technology of NUST. His research interests are in the area of millimeter-wave detection and target recognition, millimeter-wave radiometric imaging technology, millimeter- wave trajectory monitoring technology and antenna/RCS testing technology.

Prof. Xu is a Senior Member of the Chinese Institute of Electronics. $\mathrm{He}$ is a member of China Standardization Committee of Fuze and China Association of Inventions 